\title{
Die Förderung und Institutionalisierung von Komplementärmedizin in Deutschland
}

\author{
Andreas Michalsen \\ Vorstandsvorsitzender der Karl und Veronica Carstens-Stiftung, Essen, Deutschland
}

Vor 30 Jahren gab es einen gravierenden Missstand im deutschen Gesundheitssystem: Die weitgehende Ignoranz der wissenschaftlichen bzw. akademischen Medizin gegenüber der Naturheilkunde, Homöopathie und Komplementärmedizin. Die Gründer der Karl und Veronica Carstens-Stiftung - der damalige Bundespräsident Karl Carstens und seine Frau Veronica Carstens - hatten es sich zur Aufgabe gemacht, darin Einfluss zu nehmen und leiteten einen Kurswechsel mit dem Ziel der «wissenschaftlichen Durchdringung von Naturheilkunde und Homöopathie» ein. 10 Jahre danach hatte dieses Programm schließlich sein wichtigstes wissenschaftliches Publikationsorgan in Deutschland bekommen: die ForsCHENDE KomplementÄRMEDIZIN. Als die Zeitschrift gegründet wurde, war die Stiftung sofort bereit, diesen Prozess zu unterstützen; denn das Anliegen der Carstens konnte ohne eine Publikationsplattform auf hohem wissenschaftlichem Niveau nicht gelingen. Die Forschende Komplementärmedizin wurde diesem Anspruch mit ihrem Gutachtersystem gerecht und war eine der beiden ersten in MEDLINE/PubMed gelisteten Fachzeitschriften im Bereich der komplementären Heilverfahren in Europa. Sie war zudem das erste Periodikum zur Komplementärmedizin, in dem auch in deutscher Sprache publiziert werden konnte. Das ist insofern wichtig, als dass im deutschsprachigen Raum eine reichhaltige Tradition der Naturheilkunde existiert und Methoden bzw. Standards verankert sind, die in anderen Ländern weniger bekannt oder nicht möglich sind.

Zahlreiche Vorstandsmitglieder, Mitarbeiter oder Projektleiter der Stiftung waren oder sind als Editors oder im Editorial Board der Forschenden KomplementÄRMEDIZIN aktiv wie z.B. Harald Walach, Klaus Linde, Claudia Witt, Gustav Dobos, Frauke Musial, Jost Langhorst, Rainer Lüdtke und viele weitere. Die Zeitschrift stellt für die Karl und Veronica Carstens-Stiftung eine wichtige Publikationsplattform dar: Im Laufe der Jahre wurden mehr als 30 Originalarbeiten aus Projekten und der Nachwuchsförderung veröffentlicht. Zusätz- lich waren die Mitarbeiter der Stiftung bei fast 20 Arbeiten, die in der Zeitschrift publiziert wurden, Autoren oder Koautoren.

Wie ist die Situation der Komplementärmedizin heute? Welche Etappenziele wurden erreicht? Welche stehen noch aus?

Die Nachfrage bezüglich der Naturheilkunde ist ungebrochen: Mehr als 60\% der Deutschen nehmen Verfahren der Komplementärmedizin in Anspruch. Die Anzahl der publizierten Fachartikel weltweit ist in den vergangenen Jahren sprunghaft angestiegen. Die Evidenz zu einigen Therapieverfahren wie z.B. der Akupunktur ist inzwischen beachtlich, auch im Vergleich zu konventionellen medizinischen Methoden. Ist die Vision von Karl und Veronica Carstens also wahr geworden? Ist die Integration in die Wissenschaft gelungen? Ja, aber sie ist fragil; denn während niedergelassene Ärzte für Naturheilverfahren, Homöopathie, manuelle Medizin, und Akupunktur einen festen Stand haben, sind die universitäre und akademische Naturheilkunde und Komplementärmedizin zarte Pflänzchen in Deutschland. Trotz aller versorgungspolitischer Relevanz, wachsender Evidenz und Datenlage gibt es immer noch keine Wissenschaftsförderung aus öffentlichen Mitteln. Die bestehenden Professuren und Lehrstühle sind aus Drittmitteln finanziert. Die Forschung wird von einzelnen Stiftungen und Unternehmen unterstützt und die Drittmittelförderung ist gegenüber dem konventionellen Segment insgesamt marginal. Nicht zuletzt sind Naturheilkunde und Komplementärmedizin nur vereinzelt in die stationäre und ambulante Regelversorgung der großen Universitätskliniken und Lehrkrankenhäuser eingebunden. Bei der flächendeckenden Translation in die klinische Versorgung stehen wir also erst am Anfang. Wiederholt ist die Erfahrung gemacht worden, dass auch wissenschaftlich gut belegte Verfahren, wie zuletzt Akupunktur, Blutegeltherapie, Yoga und andere, dennoch nicht in der Klinik und Praxis angemessen berücksichtigt und implementiert werden. Von einer Grundversorgung, die

\section{KARGER \\ Fax +497614520714 \\ Information@Karger.com}

www.karger.com (c) 2013 S. Karger GmbH, Freiburg

1661-4119/13/0201-0013\$38.00/0

Accessible online at:

www.karger.com/fok
Prof. Dr med. Andreas Michalsen

Karl und Veronica Carstens-Stiftung

Am Deimelsberg 36, 45276 Essen, Deutschland

info@carstens-stiftung.de 
Komplementärmedizin beinhaltet, sind wir noch weit entfernt. Naturheilkunde und Komplementärmedizin stehen heute zumeist nur denjenigen zur Verfügung, die darüber Bescheid wissen, nachfragen, sie einfordern und sie sich gleichzeitig leisten können.

Die weitere Zukunft der Naturheilkunde und Komplementärmedizin wird vermutlich nicht nur von wissenschaftlicher Evidenz, sondern auch maßgeblich von politischen und wirtschaftlichen Kontextfaktoren abhängen. Mit der Umstellung ihrer Förderpolitik zugunsten einer operativen Strategie nimmt die Karl und Veronica Carstens-Stiftung diese neue Herausforderung an, indem sie eigene Projekte gestaltet, deren Ergebnisse einen direkten Bezug zur Versorgung haben, modellhaft sind und einen exzellenten methodischen Anspruch verfolgen. Beispiele hierfür sind ein Forschungsprogramm zu den Effekten von therapeutischer Blutentnahme
(Aderlass) und Blutspende, interdisziplinäre Expertenkonferenzen zur Geriatrie und ein einzigartiges Nachwuchsprogramm. Zudem stellt die Karl und Veronica Carstens-Stiftung ausgewählten Universitätsbibliotheken ein kostenloses Abonnement der ForsCHENDEN KoMPLEMENTÄRMEDIZIN zur Verfügung, insbesondere, damit Studierende auch in Zeiten knapper Bibliotheksbudgets auf Fachbeiträge zur Komplementärmedizin zugreifen können.

Bei der Veröffentlichung der Ergebnisse aus den Projekten der Stiftung wird die ForsChende KomplementärmedizIn weiterhin ein wichtiger Partner sein. Für die wissenschaftsgestützte Komplementärmedizin in Europa ist sie das federführende Organ. Stellvertretend für den Vorstand der Karl und Veronica Carstens-Stiftung danke ich dem Karger Verlag für das Engagement und wünsche der Zeitschrift alles Gute für die Zukunft. 